\title{
455 - Face to face vs. online cognitive stimulation for people with cognitive impairment. A controlled trial.
}

Authors:

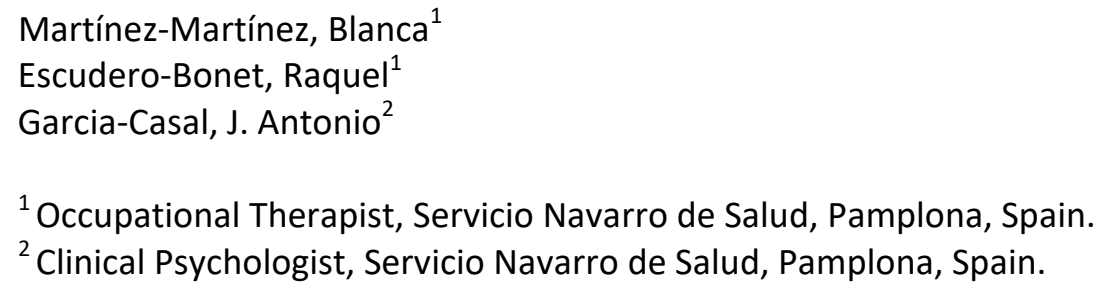

It's been proved that cognitive stimulation has direct effects over the improvement of general cognitive functions in people with cognitive impairment. People older than 50 are progressively familiar with computers and mobile devices, opening an opportunity for computer based online cognitive stimulation programmes.

\section{Objectives}

To compare the efficacy of face to face cognitive stimulation (FFCS) with online home delivered cognitive stimulation (OCS) regarding adherence, number of sessions, cognitive function and mood.

Patients and Methods

Participants were enrolled in a public memory clinic; 51 patients with cognitive decline were assigned to two groups: 27 received FFCS in a group format and 24 received OCS with the assistance of a carer. Both face to face and online interventions where designed and supervised by a trained Occupational Therapist. Pre and post assessments were carried out by a Clinical Psychologist with the Mini Mental State Exam (MMSE), the clock test, and the brief Geriatric Depression Scale. No differences were found between groups in age (69.65 \pm 9.74 years), cognitive function (MMSE $=24.63 \pm 3.67)$, gender (55\% women) and education. The treatment consisted of 32 sessions of CS held twice a week during 4 months.

\section{Results}

Data was analysed with nonparametric statistics and between group effect sizes were calculated. The FFCS participants completed $29.19 \pm 1.73$ sessions while the OCS group completed $26.00 \pm 10.64$ sessions $(p<.000)$. All the participants in the FFCS group $(100 \%)$ and 14 of the OCS group $(58 \%)$ finished the treatment $(p<.000)$.

Between group effect size favoured the FFCS intervention for MMSE $\left(d_{c}=0.36\right)$. No between group differences were found for mood $\left(d_{c}=-0.5\right)$ or the clock test $\left(d_{c}=0.13\right)$.

Conclusions

FFCS is better accepted by patients than OCS, with higher rates of adherence and less dropouts. FFCS leads to better results in the preservation of cognitive capacity.

Key words. Cognitive Stimulation. Online. Computer based. Cognitive impairment. 